\title{
Nurses' perceptions of standardised assessment and prevention of complications in an ICU
}

\begin{abstract}
Aims and objectives: To describe nurses' perceptions of evidence-based recommendations to prevent complications in a Malaysian intensive care unit. Background: Ventilator-associated pneumonia, catheter-related blood stream infection and pressure ulcer are three frequent adverse events in the intensive care unit. Implementing evidenced-based practice is critical in prevention of these complications. Design: A qualitative focus group study. Methods: Focus groups were conducted with nurses in the intensive care unit of a regional hospital in Malaysia following evidence-based interventions. Focus group transcripts were analysed using the method of thematic analyses. Results: Thirty-four nurses participated in eight focus groups. The main themes derived from the interviews: (1) nurses' knowledge impacts on the change process; (2) initial resistance, ambivalence and movement to acceptance; and (3) hierarchical organisational structure can hinder the change process. Conclusion: Enhancing nurses' knowledge and attitudes of evidence-based practice, providing them with tools to monitor their clinical practice, and empowering them to change practice are likely to be important in influencing clinical outcomes. Increasing the emphasis on evidence-based practice in nursing curricula and engaging in cultural change processes in the workplace are necessary to improve clinical outcomes. Relevance to clinical practice: These findings provide valuable information for implementing clinical practice improvement interventions.
\end{abstract}

Keyword: Clinical practice improvement; Intensive care unit; Malaysia; Nurses 